\title{
Multiferroicity Induced by Dislocated Spin-Density Waves
}

\author{
Joseph J. Betouras, ${ }^{1,2}$ Gianluca Giovannetti, ${ }^{2,3}$ and Jeroen van den Brink ${ }^{2,4}$ \\ ${ }^{1}$ School of Physics and Astronomy, Scottish Universities Physics Alliance, University of St. Andrews, North Haugh KY16 9 SS, \\ United Kingdom \\ ${ }^{2}$ Instituut-Lorentz for Theoretical Physics, Leiden University, P.O. Box 9506, 2300 RA Leiden, The Netherlands \\ ${ }^{3}$ Faculty of Science and Technology, University of Twente, P.O. Box 217, 7500 AE Enschede, The Netherlands \\ ${ }^{4}$ Institute for Molecules and Materials, Radboud Universiteit Nijmegen, P.O. Box 9010, 6500 GL Nijmegen, The Netherlands
}

(Received 12 February 2007; published 20 June 2007)

\begin{abstract}
We uncover a new pathway towards multiferroicity, showing how magnetism can drive ferroelectricity without relying on inversion symmetry breaking of the magnetic ordering. Our free-energy analysis demonstrates that any commensurate spin-density-wave ordering with a phase dislocation, even if it is collinear, gives rise to an electric polarization. Because of the dislocation, the electronic and magnetic inversion centers do not coincide, which turns out to be a sufficient condition for multiferroic coupling. The novel mechanism explains the formation of multiferroic phases at the magnetic commensurability transitions, such as the ones observed in $\mathrm{YMn}_{2} \mathrm{O}_{5}$ and related compounds. We predict that in these multiferroics an oscillating electrical polarization is concomitant with the uniform polarization. On the basis of our theory, we put forward new types of magnetic materials that are potentially ferroelectric.
\end{abstract}

DOI: $10.1103 /$ PhysRevLett.98.257602

PACS numbers: 77.80.-e, 75.30.Fv, 75.47.Lx

Introduction. - The recent discovery of novel materials in which magnetic and ferroelectric order can coexisttermed multiferroics - has sparked a new surge of interest in this field [1-11]. From a technological point of view, the possibility to control magnetic properties by electric fields and, vice versa, ferroelectric order by magnetic fields, is very desirable. By definition multiferroics, sometimes also called magnetoelectrics, possess at least two switchable states of electric polarization $(\mathbf{P})$ and magnetization $(\mathbf{M})$. Although there is a number of materials that exhibit both ferroelectricity and magnetism, it might come as a surprise that there need not necessarily be a large coupling between them. Typically, the ferroelectric transition temperature is much higher than the magnetic one, and a coupling between the two order parameters is weak. Representative examples of this behavior are the transition metal perovskites $\mathrm{BiFeO}_{3}$ and $\mathrm{BiMnO}_{3}$ [12-15].

For potential applications of multiferroics, it is very important to establish general mechanisms that give rise to a coupling of ferroelectricity and magnetism. More specifically, one would like to construct situations where the two ordering temperatures can become close or even coincide: In that case, one expects strong multiferroic behavior with a pronounced interdependence of the ferroelectric and magnetic order parameters. This then directly opens up the possibility to switch the macroscopic ferroelectric polarization by applying an external magnetic field, which is the key to future applications of multiferroic materials.

In this Letter, we report a novel route for potential strong coupling multiferroicity. A straightforward GinzburgLandau free-energy analysis demonstrates that spin-density-wave (SDW) ordering with a phase dislocation gives rise to an electric polarization. A remarkable feature is that in such a SDW a commensurate oscillating electric polarization causes a net uniform ferroelectric moment and that incommensurate ordering does not. Therefore, our mechanism explains the formation of multiferroic phases at the magnetic commensurability transitions, such as the one observed in $\mathrm{YMn}_{2} \mathrm{O}_{5}$-indeed, a dislocated SDW material with an acentric magnetization. Based on this novel mechanism, we will also suggest new types of multiferroic materials.

From a theoretical point of view, the question of possible magnetoelectric couplings is best addressed within the framework of the Landau theory of phase transitions, since it is based on symmetry considerations alone. In this sense, it captures the essence of any microscopic mechanism for the coupling between ferroelectricity and magnetism [16]. Such a Landau analysis has already uncovered a case in which ferroelectricity is induced by magnetic ordering. This is the case when the magnetic ordering is of a type that breaks chiral symmetry. This is realized in spiral or helical spin-density-wave systems [17-21]. For instance, $\mathrm{TbMnO}_{3}, \mathrm{DyMnO}_{3}, \mathrm{Ni}_{3} \mathrm{~V}_{2} \mathrm{O}_{8}$, and the recently discovered multiferroic $\mathrm{MnWO}_{4}$ all fall in this class [3,12,19,22-24]. "Ferroelectricity generated by magnetic chirality" has established itself as the leading paradigm for both theoretical and experimental investigations in the field of multiferroics with magnetically induced ferroelectricity.

However, a "rule" that chiral symmetry needs to be broken in order to induce a ferroelectric moment at a magnetic phase transition is questionable. First of all, there are notable exceptions, in particular, the manganites $R \mathrm{Mn}_{2} \mathrm{O}_{5}$ ( $R$ is a rare earth, e.g., Tb, Ho, Dy, or Y). Experiment shows that in these materials a ferroelectric polarization emerges that is due to collinear magnetic ordering with no indication of magnetic chiral symmetry breaking 
$[25,26]$. Second, in several of these systems, the ferroelectric polarization appears spontaneously at magnetic commensurate-incommensurate transitions. This has even lead to the claim that — on empirical grounds - a commensurate spin state be essential to the ferroelectricity in multiferroic $R \mathrm{Mn}_{2} \mathrm{O}_{5}$ [27]. Such an empirical rule cannot be explained within the theoretical framework of chiral symmetry breaking. Instead, as we show below that this behavior is inherent to a novel type of multiferroics which displays a strong interdependence of magnetization and ferroelectric polarization: materials with a dislocated, acentric spin-density-wave ordering.

Free-energy analysis. - We prove the above assertions by considering a continuum field theory of the Landau type that incorporates at the same time ferroelectric polarization $\mathbf{P}$ and magnetic SDW order $\mathbf{M}(\mathbf{r})$, where $\mathbf{r}$ is the spatial coordinate. The form of the coupling between the electric polarization $\mathbf{P}(\mathbf{r})$ and magnetization $\mathbf{M}(\mathbf{r})$ can be found from general symmetry arguments [17,18]. First, time reversal $t \rightarrow-t$ should leave the magnetoelectric coupling invariant. As it transforms $\mathbf{M} \rightarrow-\mathbf{M}$ and leaves $\mathbf{P}$ invariant, time reversal requires that the lowest order coupling has to be quadratic in $\mathbf{M}$. The symmetry of spatial inversion, i.e., $\mathbf{r} \rightarrow-\mathbf{r}$, which sends $\mathbf{P}$ to $-\mathbf{P}$, is respected when the coupling of a homogeneous polarization to an inhomogeneous magnetization is linear in $\mathbf{P}$ and contains one gradient of $\mathbf{M}$. These symmetry considerations lead to a magnetoelectric coupling term in the Landau free energy of the form

$$
\begin{aligned}
F_{\mathrm{ME}}(\mathbf{r})= & \mathbf{P} \cdot\left\{\gamma \nabla\left(\mathbf{M}^{2}\right)+\gamma^{\prime}[\mathbf{M}(\nabla \cdot \mathbf{M})-(\mathbf{M} \cdot \nabla) \mathbf{M}]\right. \\
& +\cdots\} .
\end{aligned}
$$

The first term on the right-hand side is proportional to the total derivative of the square of the magnetization. It gives a surface contribution only when the free energy is integrated over the spatial coordinates and the polarization $\mathbf{P}$ is assumed to be independent of $\mathbf{r}[17,18]$. It is central to our physical ideas not to make this assumption.

It is easy to show that the second term in the equation above, proportional to $\gamma^{\prime}$, is nonzero only if the magnetization $\mathbf{M}$ breaks chiral symmetry, which is the canonical route towards a strong dependence of $\mathbf{P}$ on $\mathbf{M}$. On physical grounds, this term can readily be understood. In a ferroelectric, inversion symmetry is broken as the system sustains a macroscopic polarization $\mathbf{P}$ that is pointing into a particular direction in space. Therefore, this polarization can only couple to the magnetization if and only if $\mathbf{M}$ also has a directionality and lacks a center of inversion symmetry. One immediately understands that this occurs when the magnetization is spiraling along some axis. This also implies, vice versa, that the chiral magnetic ordering induces a ferroelectric polarization. Recently, this situation was considered in detail [18]; we do not consider it here, set $\gamma^{\prime}=0$ in the following, and consider only the case of nonzero $\gamma$.
As our focus is on systems that in the absence of magnetism show no instability to ferroelectricity, we take into account only the quadratic term in $\mathbf{P}$ in the electric part of the free energy:

$$
F_{E}(\mathbf{r})=\frac{\mathbf{P}(\mathbf{r})^{2}}{2 \chi_{E}(\mathbf{r})}
$$

where $\chi_{E}(\mathbf{r})$ is the dielectric susceptibility. From the variation of $F_{\mathrm{ME}}(\mathbf{r})+F_{E}(\mathbf{r})$ with respect to $\mathbf{P}(\mathbf{r})$ for a given magnetization, one can find the value of the static polarization field. It is important to note that the polarization is due to all charges in the system: the atomic nuclei in the lattice plus the electrons. Magnetization, instead, is a density wave of the spin of the electrons alone. The dependence of the fields $\mathbf{P}(\mathbf{r})$ and $\mathbf{M}(\mathbf{r})$ on their spatial coordinates $\mathbf{r}=(x, y, z)$ need not be the same, of course. As we will consider an acentric SDW ordering, we explicitly keep the spatial dependence of the dielectric susceptibility in the equation above. For simplicity, we shall keep only the $x$ dependence, as our results are readily generalized to include the other spatial dependencies.

Dislocated SDW.-The magnetization of a phasedislocated, acentric SDW is given by $M=M_{0} \cos \left(q_{m} x+\right.$ $\phi)$, where $q_{m}$ is the magnetic ordering wave vector and $\phi$ its phase. The fact that the phase of the magnetization is dislocated implies that $\phi$ is finite: The magnetization is shifted with respect to the electronic density. Hence, the magnetization is also phase-dislocated with respect to the lattice. However, as the magnetization is collinear and sinusoidal, it obviously has a center of symmetry and no directionality. Thus, on the basis of the arguments that exist prior to the present work, one would be led to conclude that it does not couple to $\mathbf{P}$. Such a conclusion, however, would be incorrect. In our theory for multiferroicity, we indeed assume both the electronic or lattice structure and the magnetic ordering to be inversion invariant, but, due to the dislocation, the electronic and magnetic inversion centers do not coincide. The implies that magnetization and polarization together break inversion symmetry, which turns out to be a sufficient condition for multiferroic coupling. This underlines the interdependence of the lattice and magnetic structure for generating a ferroelectric moment.

The expression of the free energy can be simplified by first introducing in Eq. (2) the Fourier components of the inverse electronic susceptibility [we need only and keep the first two: $\chi_{E}^{-1}=e_{0}+e_{1} \cos (q x)$, which is assumed to be positive definite] and by using the ansatz $P=p_{0}+$ $p_{1} \cos (q x)$. Note that the oscillating part of the polarization, proportional to $p_{1}$, does not contribute to a net ferroelectric moment as its spatial average vanishes. However, its presence is crucial, as this oscillating polarization couples directly to the gradient of the magnetization squared and, at the same time, to the uniform polarization $p_{0}$. 
Electric polarizations. - We integrate the free energy over its spatial coordinates and minimize it with respect to $p_{0}$ and $p_{1}$. This directly leads to our main result for the homogenous ferroelectric polarization:

$$
p_{0}=\frac{-\gamma q_{m} M_{0}^{2}}{2} \frac{e_{1}}{2 e_{0}^{2}-e_{1}^{2}} \sin 2 \phi .
$$

We also find a concomitant oscillating polarization in the ferroelectric equilibrium state

$$
p_{1}=-2 e_{0} p_{0} / e_{1},
$$

which is particular to our magnetoelectric coupling mechanism. These expressions hold under the condition that $q_{m}=q / 2$; i.e., the magnetic wave vector is commensurate and equal to half of the lattice wave vector. The polarization vanishes for the case that $q_{m}$ is incommensurate. These relations between the two ordering wave vectors $q$ and $q_{m}$ are due to the fact that the spatial integral of the free-energy terms of the kind $\int \cos (q x) \cos \left(q^{\prime} x\right) d x$ vanishes unless $q=$ $q^{\prime}$. From the expression above, it is clear that a finite dislocation of the SDW with respect to the electronic density - a nonzero value of $\phi$-is essential to produce a nonzero macroscopic ferroelectric moment [28].

The physical explanation for the resulting ferroelectricity is elementary. As mentioned above, our acentric magnetization density $\mathbf{M}^{2}$ is sinusoidal and by itself inversion invariant (we can always choose an appropriate origin of the coordinate system). Therefore, it does not possess an absolute directionality. However, in an acentric SDW system, $\mathbf{M}^{2}$ is lagging behind somewhat with respect to the polarization $\mathbf{P}$, which is the immediate consequence of the finite phase difference $\phi$. Thus, we conclude that $\mathbf{M}^{2}$ has a directionality relative to $\mathbf{P}$, which is a sufficient condition for a direct coupling between the two order parameters and the emergence of a macroscopic ferroelectric polarization in the acentric SDW systems.

Application to materials. - The above analysis can be directly applied to the above mentioned class of multiferroic compounds $R \mathrm{Mn}_{2} \mathrm{O}_{5}$. These insulators order antiferromagnetically below $45 \mathrm{~K}$ and display a complex sequence of incommensurate (ICM) and commensurate (CM) magnetic phases and no sign of magnetic chirality. However, it has been known for a long time that, for instance, $\mathrm{YMn}_{2} \mathrm{O}_{5}$ displays acentric SDW ordering and significant magnetoelastic effects [26,29]. The acentricity of the SDW is due to the frustration of magnetic order [26]. From our calculations, one expects a ferroelectric polarization to appear if the acentric SDW ordering is commensurate, with a polarization that is along the direction of $\nabla(\mathbf{M})^{2}$. Indeed, experimentally in $\mathrm{YMn}_{2} \mathrm{O}_{5}$ a spontaneous ferroelectric polarization along the crystallographic $b$ axis appears when commensurate spin ordering sets in below $45 \mathrm{~K}$. Most remarkably, at $23 \mathrm{~K}$ a magnetic CM to ICM transition takes place. At this transition, the magnitude of the magnetization does not change, but the ferroelectric polarization collapses, in perfect agreement with our theoretical considerations.

The theoretical observation that ferroelectricity depends directly on the commensurability of the dislocated SDW is also supported by thermal expansion data for $R=\mathrm{Ho}$, Dy, and $\mathrm{Tb}$. These show that also the high temperature ferroelectric transition around $45 \mathrm{~K}$ coincides with a lock-in of the magnetic wave vector from incommensurate to commensurate values [30]. In $\mathrm{HoMn}_{2} \mathrm{O}_{5}$, moreover, at the low temperature ferroelectric transition a magnetic field induces ICM-CM transitions that go hand in hand with the appearance of macroscopic polarization [27]. All of these experimental data are in accordance with the acentric SDW route to multiferroicity that we propose in this Letter. Note that in ICM phases one can expect some residual ferroelectricity to appear when the system is partially commensurable or if domain formation occurs.

Another class of systems to which our analysis applies is the perovskite manganites, e.g., $\operatorname{Pr}_{1-x} \mathrm{Ca}_{x} \mathrm{MnO}_{3}$. For a large doping concentration, a number of different charge, spin, and orbital ordered states are predicted and found [31,32]. In particular, there is experimental evidence for the existence of a phase of the system in which the magnetic moment is centered on $\mathrm{Mn}-\mathrm{Mn}$ bonds [33]. Theoretically, an acentric SDW is found to be the magnetic ground state when $0.4<x<0.5$, and, indeed, instability of the lattice towards a polar ferroelectric distortion is expected and found [7,34]. In single crystals of another member of the same class of materials, $\mathrm{Gd}_{1-x} \mathrm{Sr}_{x} \mathrm{MnO}_{3}$ electric polarization has been recently observed as well [35], and it is to be attributed to the same physics.

So the present novel magnetoelectric coupling explains the observed multiferroic behavior in two classes of ceramic materials. Our theory suggests that a third, completely different class of systems can show this behavior: organic charge transfer salts. Quasi-one-dimensional molecular crystals (TMTTF) $)_{2} \mathrm{Br}$, (TMTSF) $)_{2} \mathrm{PF}_{6}$, and $\alpha$-(BEDT-TTF $)_{2} M \mathrm{Hg}(\mathrm{SCN})_{4}$ show an unusual coexistence of spin- and charge-density waves [36]. As in the above mentioned perovskite manganites, such a superposition of spin- and charge-density-wave orderings produces an acentric magnetization. The fact that our conditions of acentricity and commensuration are fulfilled makes the organic charge transfer salts, from the perspective of our theory, candidates par excellence to exhibit multiferroic behavior. The strength of the induced polarization will depend on the microscopic details of these systems. For a reliable estimate, one will need to go beyond the phenomenological Ginzburg-Landau approach that we have presented here [37].

Conclusions. - We have shown how magnetism can drive ferroelectricity without relying on inversion symmetry breaking of the magnetic ordering. Starting from the Landau-Ginzburg theory, we have revealed a new mechanism that leads to magnetoelectric coupling in SDW sys- 
tems. The two key ingredients are the finite dislocation and the commensuration of the SDW with respect to the lattice. There are at least two classes of materials that exhibit this behavior and are thus understood in the light of the present theory. Besides explaining the observed magnetoelectric coupling in these materials, we also predict that in these systems an oscillating electrical polarization is concomitant with the uniform polarization and that the magnetic ordering wave vector is half the electronic one. Furthermore, on the basis of our theory, we suggest that magnetoelectric coupling occurs in a class of materials that was little considered so far, the organic charge transfer salts, opening up a general pathway to construct new multiferroic materials.

We thank Maxim Mostovoy, Tsuyoshi Kimura, Claude Pasquier, Laurent Chapon, Paolo Radaelli, and Jan Zaanen for stimulating discussions. This work is supported by FOM and the Dutch Science Foundation.

[1] W. Eerenstein, N.D. Mathur, and J.F. Scott, Nature (London) 442, 759 (2006).

[2] S.-W. Cheong and M. Mostovoy, Nat. Mater. 6, 13 (2007).

[3] T. Kimura et al., Nature (London) 426, 55 (2003).

[4] N. Hur et al., Nature (London) 429, 392 (2004).

[5] T. Lottermoser et al., Nature (London) 430, 541 (2004).

[6] B. B. van Aken, T. T. M. Palstra, A. Filippetti, and N. A. Spaldin, Nat. Mater. 3, 164 (2004).

[7] D. V. Efremov, J. van den Brink, and D. I. Khomskii, Nat. Mater. 3, 853 (2004).

[8] D. V. Efremov, J. van den Brink, and D. I. Khomskii, Physica (Amsterdam) 359B, 1433 (2005).

[9] J. Hemberger et al., Nature (London) 434, 364 (2005).

[10] C. Ederer and N. A. Spaldin, Nat. Mater. 3, 849 (2004).

[11] R. E. Cohen, Nature (London) 358, 136 (1992).

[12] D. I. Khomskii, J. Magn. Magn. Mater. (to be published); arXiv:cond-mat/0601696.

[13] N. A. Hill, Annu. Rev. Mater. Res. 32, 1 (2002).

[14] C. Ederer and N. A. Spaldin, Phys. Rev. B 71, 060401(R) (2005).

[15] A. M. dos Santos et al., Solid State Commun. 122, 49 (2002).
[16] P. Chandra and P. B. Littlewood, arXiv:cond-mat/0609347.

[17] I. E. Dzyaloshinskii, Sov. Phys. JETP 10, 628 (1960).

[18] M. Mostovoy, Phys. Rev. Lett. 96, 067601 (2006).

[19] G. Lawes et al., Phys. Rev. Lett. 95, 087205 (2005).

[20] I. A. Sergienko and E. Dagotto, Phys. Rev. B 73, 094434 (2006).

[21] H. Katsura, N. Nagaosa, and N. V. Balatsky, Phys. Rev. Lett. 95, 057205 (2005).

[22] T. Goto, T. Kimura, G. Lawes, A.P. Ramirez, and Y. Tokura, Phys. Rev. Lett. 92, 257201 (2004).

[23] T. Kimura, G. Lawes, T. Goto, Y. Tokura, and A. P. Ramirez, Phys. Rev. B 71, 224425 (2005).

[24] K. Taniguchi, N. Abe, T. Takenobu, Y. Iwasa, and T. Arima, Phys. Rev. Lett. 97, 097203 (2006); O. Heyer et al., J. Phys. Condens. Matter 18, L471 (2006).

[25] G. R. Blake et al., Phys. Rev. B 71, 214402 (2005).

[26] L. C. Chapon, P. G. Radaelli, G. R. Blake, S. Park, and S. W. Cheong, Phys. Rev. Lett. 96, 097601 (2006).

[27] H. Kimura et al., arXiv:cond-mat/0602226.

[28] A nonzero value of $\phi$ can be easily predicted for, e.g., a simple Heisenberg magnet when the exchange interactions with frustrating exchange couplings. More details will be reported elsewhere [J.J. Betouras, G. Giovannetti, and J. van den Brink (to be published)].

[29] C. Wilkinson, F. Sinclair, P. Gardner, J. B. Forsyth, and B. M. R. Wanklyn, J. Phys. C 14, 1671 (1981).

[30] C. R. dela Cruz et al., Phys. Rev. B 73, 100406(R) (2006).

[31] J. van den Brink and D. Khomskii, Phys. Rev. Lett. 82, 1016 (1999); J. van den Brink, G. Khaliullin, and D. Khomskii, Phys. Rev. Lett. 83, 5118 (1999); 86, 5843 (2001); J. van den Brink and D. Khomskii, Phys. Rev. B 63, 140416(R) (2001).

[32] For a review, see J. van den Brink, G. Khaliullin, and D. Khomskii, in Colossal Magnetoresistive Manganites, edited by T. Chatterji (Kluwer Academic, Dordrecht, 2004).

[33] A. Daoud-Aladine, J. Rodriguez-Carvajal, L. PinsardGaudart, M.T. Fernandez-Diaz, and A. Revcolevschi, Phys. Rev. Lett. 89, 097205 (2002).

[34] F. Krüger et al. (to be published).

[35] A. M. Kadomtseva et al., JETP Lett. 82, 590 (2005).

[36] S. Mazumdar, S. Ramasesha, R. T. Clay, and D. K. Campbell, Phys. Rev. Lett. 82, 1522 (1999).

[37] G. Giovannetti and J. van den Brink (to be published). 